\title{
Host and symbiont genetic contributions to fitness in a Trichogramma-Wolbachia symbiosis
}

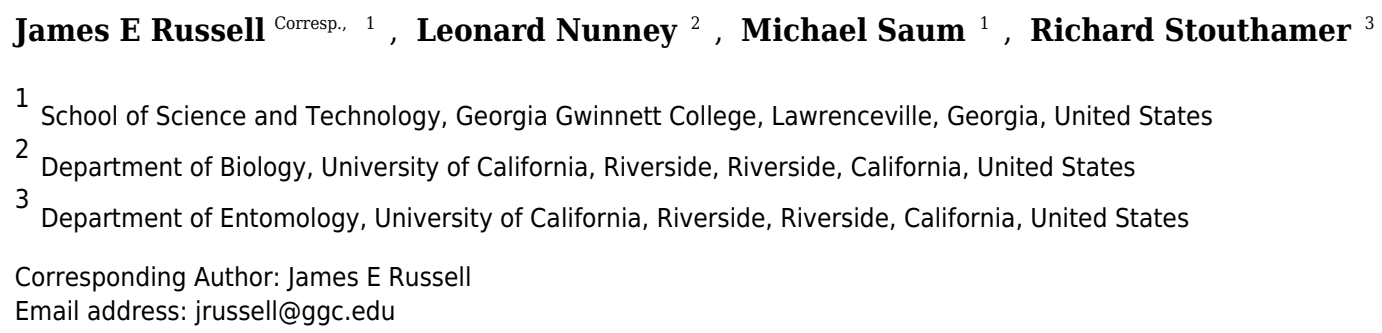

The fitness effects associated with Wolbachia infection have wide-ranging ecological and evolutionary consequences for host species. How these effects are modulated by the relative influence of host and Wolbachia genomes has been described as a balancing act of genomic cooperation and conflict. For vertically transmitted symbionts, like cytoplasmic Wolbachia, concordant host-symbiont fitness interests would seem to select for genomic cooperation. However, Wolbachia's ability to manipulate host reproductive systems and distort offspring sex ratios presents an evolutionary conflict of interest with infected hosts. In the parthenogenesis-inducing (PI) form of Wolbachia found in many haplodiploid insects, Wolbachia fitness is realized through females and is enhanced by their feminization of male embryos and subsequent parthenogenetic reproduction. In contrast, as long as Wolbachia is not fixed in a population and sexual reproduction persists, fitness for the host species is realized through both male and female offspring production. How these cooperating and competing interests interact and the relative influence of host and Wolbachia genomes were investigated in the egg parasitoid Trichogramma kaykai, where Wolbachia infection has remained at a low frequency in the field. A factorial design in which laboratory cultures of Wolbachia-infected T. kaykai were cured and re-infected with alternative Wolbachia strains was used to determine the relative influence of host and Wolbachia genomes on host fitness values. Our results suggest fitness variation is largely a function of host genetic background, except in the case of offspring sex ratio where a significant interaction between host and Wolbachia genomes was found. We also find a significant effect associated with the horizontal transfer of Wolbachia strains, which we discuss in terms of the potential for coadaptation in PI Wolbachia symbioses. 
1 Host and symbiont genetic contributions to fitness in a Trichogramma-Wolbachia symbiosis.

2

3

4

5

$6 \quad{ }^{1}$ School of Science and Technology

7 Georgia Gwinnett College

8 Lawrenceville, GA 30043 USA

9

10

11

12

13

14

15

16

17

18

19

20

21

22

23

24

25

26

27

28

29

30

31

32

33

34

35

Lawrenceville, GA 30043
${ }^{2}$ Department of Biology

University of California

Riverside, CA 92521 USA

${ }^{3}$ Department of Entomology

University of California

Riverside, CA 92521 USA

Corresponding author:

James E. Russell

School of Science and Technology

Georgia Gwinnett College

Tel. 6786825669

Fax. 6784075938

Email: jrussell@ggc.edu

Running title: Trichogramma-Wolbachia coevolution 


\section{Abstract.}

The fitness effects associated with Wolbachia infection have wide-ranging ecological and evolutionary consequences for host species. How these effects are modulated by the relative influence of host and Wolbachia genomes has been described as a balancing act of genomic cooperation and conflict. For vertically transmitted symbionts, like cytoplasmic Wolbachia, concordant host-symbiont fitness interests would seem to select for genomic cooperation. However, Wolbachia's ability to manipulate host reproductive systems and distort offspring sex ratios presents an evolutionary conflict of interest with infected hosts. In the parthenogenesisinducing (PI) form of Wolbachia found in many haplodiploid insects, Wolbachia fitness is realized through females and is enhanced by their feminization of male embryos and subsequent parthenogenetic reproduction. In contrast, as long as Wolbachia is not fixed in a population and sexual reproduction persists, fitness for the host species is realized through both male and female offspring production. How these cooperating and competing interests interact and the relative influence of host and Wolbachia genomes were investigated in the egg parasitoid Trichogramma kaykai, where Wolbachia infection has remained at a low frequency in the field. A factorial design in which laboratory cultures of Wolbachia-infected T. kaykai were cured and re-infected with alternative Wolbachia strains was used to determine the relative influence of host and Wolbachia genomes on host fitness values. Our results suggest fitness variation is largely a function of host genetic background, except in the case of offspring sex ratio where a significant interaction between host and Wolbachia genomes was found. We also find a significant effect associated with the horizontal transfer of Wolbachia strains, which we discuss in terms of the potential for coadaptation in PI Wolbachia symbioses. 


\section{Introduction.}

The alphaproteobactrium Wolbachia pipientis is a widely distributed cytoplasmic symbiont among arthropods and nematodes, and occupies an ecological niche distinguished by manipulation of host reproduction. For many arthropod species host reproduction is manipulated in a parasitic manner that involves distortion of host sex ratios by male-killing, feminization or parthenogenesis-induction, and by creating reproductive barriers between infected and uninfected individuals via a process referred to as cytoplasmic incompatibility (Werren 1997). Wolbachia is also considered a mutualistic obligate symbiont among nematodes where host fertility appears to be dependent upon Wolbachia infection (Pfarr \& Hoerauf 2007). The various reproductive effects associated with Wolbachia make it one of the most influential bacterial infections known with regard to the its effect on the fitness of infected host populations..

Fitness for vertically transmitted Wolbachia is ultimately linked to female host fitness since the primary mode of transmission for Wolbachia is maternal inheritance (Wolbachia resides in the cytoplasm of host reproductive cells). Hence, the effects of Wolbachia infections on different aspects of host fitness directly affect the nature of the symbioses between Wolbachia and their hosts: negative fitness effects resulting in host-symbiont conflicts if they enhance Wolbachia fitness (e.g. by increasing transmission rate); and positive fitness effects resulting in hostsymbiont cooperation. For long-term coevolved symbioses theoretical predictions and empirical results suggest selection will favor alignment of fitness interests for maternally inherited symbionts and their female hosts (Turelli 1994; Herre et al. 1999; Wade \& Goodnight 2006; Weeks et al. 2007). However, the ability to manipulate host reproductive systems by sex ratio distortion presents a potential source of conflict between Wolbachia symbionts and their hosts, since the fitness interests of sexually reproducing hosts differ from maternally inherited 
82 Wolbachia symbionts regarding offspring sex ratios, with sexual hosts gaining optimum fitness

83 through male and female offspring and Wolbachia gaining optimum fitness through female

84 offspring alone.

85 The parthenogenesis-inducing (PI) form of Wolbachia found in several haplodiploid arthropods

86 feminizes male embryos allowing infected females to produce female offspring without mating

87 (Stouthamer et al. 1990). The mechanism by which Wolbachia induces parthenogenesis varies

88 among host species from a mechanism called gamete duplication (Trichogramma kaykai and

89 other species) to a mechanism resembling apomixis in Bryobia mite species (Stouthamer \&

90 Kazmer 1994; Weeks \& Breeuwer 2001); however, in either case the end result is the

91 parthenogenetic production of infected diploid females from unfertilized eggs. PI Wolbachia

92 infection frequencies in various species and populations of wasps, thrips, and mites are

93 extremely high (Arakaki et al. 2001; Weeks \& Breeuwer 2001; Huigens \& Stouthamer 2003;

94 Pannebakker et al. 2005; Kremer et al. 2009). Though the PI form of Wolbachia does not

95 directly prevent fertilization and sexual reproduction, in populations fixed for infection, females

96 have lost the ability to fertilize eggs and reproduce sexually (Pannebakker et al. 2005). As a

97 result, such populations (and some entire species) are dependent on Wolbachia for reproduction

98 (Russell \& Stouthamer 2011; Stouthamer et al. 2010).

99 Trichogramma kaykai, a minute egg parasitoid of the Mojave Desert, is one of the rare examples

100 of a species with a low frequency PI Wolbachia infection. The infection frequency has been

101 repeatedly measured over the past 20 years with observed frequencies reaching no higher than

102 26\% infected females (Stouthamer \& Kazmer 1994; Stouthamer et al. 2001; Huigens 2003;

103 Russell 2008). Unlike populations fixed for PI Wolbachia, infected T. kaykai females are capable

104 of sexual and parthenogenetic reproduction. Infected T. kaykai females mate with uninfected 
105 males at high frequencies and high levels of heterozygosity have been observed within the 106 infected T. kaykai population (Huigens 2003; Russell 2008).

107 Given the unusual characteristics of the T. kaykai-Wolbachia symbiosis, namely, a low infection 108 frequency and facultative parthenogenesis, the fitness effects of associated with Wolbachia 109 infection in T. kaykai have been the subject of numerous studies (Huigens et al. 2004, Miura and 110 Tagami 2004, Tagami et al. 2001, Russell et al. 2016). Comparisons between Wolbachia111 infected and antibiotically cured T. kaykai have shown consistent negative fitness effects 112 associated with Wolbachia infection. Wolbachia-free treatments produce more offspring and are 113 less likely to die in pupal development (Tagami et al. 2001, Huigens et al. 2004, Russell et al. 114 2016). Wolbachia-free treatments also fertilize eggs and produce offspring sex ratios at 115 frequencies similar to what is observed in the uninfected population (Tagami et al. 2002, Russell 116 et al. 2016). The fitness costs associated with infection may be a factor in the observed low 117 infection frequency in the field, however, host limitation may mitigate the fitness costs 118 associated with infection (Lindsey and Stouthamer 2017a). How the fitness costs associated with 119 infection are attributed to host, Wolbachia, and/or host-Wolbachia interactions is currently 120 unknown for T. kaykai.

121 To investigate these relative fitness effects of host T. kaykai genetic background, Wolbachia

122 background, and horizontal transfer of Wolbachia, we used a curing/re-infection protocol. Using 123 four clonal lines of field-caught infected T. kaykai that were maintained separately in the 124 laboratory over 97 generations, we created a factorial design of the four T. kaykai $\left(T_{i}\right)$ and 125 Wolbachia $\left(W_{i}\right)$ genomes (Fig. 1) to test hypotheses related to the T. kaykai-Wolbachia 126 interaction. We addressed two main questions. First, since novel (not strictly co-inherited) cyto127 nuclear combinations are typical in the field in this species, do such combinations show fitness 
128 variation and, if so, is it driven by differences among host genomes or symbiont genomes, or by

129 their combination? Second, does horizontal transfer of PI-Wolbachia result in decreased fitness?

\section{Materials and Methods.}

131 In this study we utilized a horizontal transmission technique (Huigens et al. 2000) to factorially 132 combine the nuclear and Wolbachia genomes in four isofemale T. kaykai lines, enabling us to 133 partition the relative fitness effects of T. kaykai (host) genome, Wolbachia genome, and the 134 host/Wolbachia interaction. Most PI Wolbachia infected species are dependent on Wolbachia for 135 reproduction and cannot reproduce sexually; thus cured cultures cannot be established. The $T$. 136 kaykai system is unique among the PI Wolbachia-infected species in that cured sexual cultures 137 can be established and re-infected.

Insect cultures: Trichogramma kaykai

T. kaykai is a small hymenopteran egg parasitoid distributed throughout the Mojave Desert of southern California (Pinto et al. 1997). The Wolbachia-infected T. kaykai isofemale lines used in this study were collected from two locations. The lines $T_{1} W_{1}$ and $T_{2} W_{2}$ (where $T$ and $W$ refer to the T. kaykai and Wolbachia genomes, respectively) were collected on April $25^{\text {th }} 2004$ and May $5^{\text {th }} 2005$, respectively, off Kelbaker Road in the Kelso Dunes region of the Mojave National Preserve $\left(34^{\circ} 57^{\prime} 55.31^{\prime} \mathrm{N}, 115^{\circ} 38^{\prime} 35.75^{\prime} \mathrm{W}\right)$. Collection permits were obtained from Debra Hughson (science advisor, Mojave National Preserve, permit number: moja-2006-sci-0015) and annual reports contributed to the United States National Park Service, Mojave National Preserve. The lines $T_{3} W_{3}$ and $T_{4} W_{4}$ were collected on June $14^{\text {th }} 2003$ off California State Highway 247 near the Stoddard Ridge (34 $\left.37^{\prime} 48.52^{\prime} \mathrm{N}, 116^{\circ} 57^{\prime} 08.99^{\prime} \mathrm{W}\right)$; no specific permission was required for collection at this location. For both locations, eggs of the butterfly Apodemia mormo

150 (Riodinidae) were removed from the host plant Eriogonum inflatum (Polygonaceae), taken to the 
$151 \mathrm{lab}$, and placed individually in $1.2 \mathrm{ml}$. collection vials. The vials were incubated at $24^{\circ} \mathrm{C}, \mathrm{L}: \mathrm{D}=$

$15216: 8 \mathrm{hrs}$ and 50\% relative humidity until emergence. Parasitized $A$. mormo eggs typically contain

153 between 3 to 5 Trichogramma wasps. Several Trichogramma species have been collected from

154 A. mormo eggs in the Mojave Desert, but the dominant egg parasitoid during the time of our

155 collection was T. kaykai. T. kaykai species were identified morphologically (Pinto et al. 1997).

156 Upon emergence, broods containing male wasps were discarded, since they were presumed

157 uninfected. Females from all female broods were isolated for further testing. No field collections

158 involved handling endangered or protected species.

159 Wolbachia infection status

160 The infection status of the isolated wasps was first established by virgin production of daughters

161 and confirmed by amplifying 16S rDNA characteristic of Wolbachia. To produce offspring, the

162 isolated virgin females were given Ephestia kuehniella host eggs attached to cardstock paper

163 with double-sided tape (eggcards). If the resulting F1 offspring were mostly female the line was

164 maintained in the laboratory as an infected isofemale culture. All lines used in this study

165 produced highly female-biased offspring sex ratios as virgins, producing some males as they

166 aged. This is a pattern typical of Wolbachia infected T. kaykai females (Hohmann et al. 2001;

167 Miura \& Tagami 2004). Consistent with this parthenogenesis, the lines also tested positive for

168 Wolbachia infection, based on $16 \mathrm{~S}$ rDNA. The 16S rDNA was amplified using PCR primers

169 specific for Wolbachia: 16sBf 5' -TTCGGCCGGATTTTACACAA- 3', 16sBr 5'-

170 AGGGATTAGCTTAGGCTTG- 3' (Werren et al. 1995), using a thermocycling protocol of:

171 initial denaturation at $94^{\circ} \mathrm{C}$ for $2 \mathrm{~min}$, followed by 38 cycles at $94^{\circ} \mathrm{C}$ for $30 \mathrm{~s}, 55^{\circ} \mathrm{C}$ for $50 \mathrm{~s}, 72^{\circ}$

$172 \mathrm{C}$ for $1 \mathrm{~min} 30 \mathrm{~s}$; and a final extension at $72^{\circ} \mathrm{C}$ for $10 \mathrm{~min}$. Four Wolbachia MLST loci were

173 used for typing the strains used in this study (Baldo et al. 2006). No variation among the 
174 Wolbachia types from the four host lines was observed for these four loci. The coxA, gatB, and 175 hcpA MLST loci in T. kaykai matched strain type 486 in the Wolbachia MLST database. There

176 was no strain type match for the T. kaykai fbpA locus; this locus shared sequence similarity with

$177 f b p A$ sequence 359 , with 4 sequence differences.

178 Curing of infected lines.

179 The two experimental lines, $T_{1} W_{1}, T_{2} W_{2}$, were maintained in laboratory cultures for 138

180 generations and the two experimental lines, $T_{3} W_{3}, T_{4} W_{4}$, were maintained for 97 generations after

181 initial isofemale isolation from field collected samples. After this period each of the lines was

182 subdivided into an infected and a cured culture. Cured cultures were obtained following three

183 generations of antibiotic treatment. This involved feeding newly emerged infected females a 5

$184 \mathrm{mg} / \mathrm{ml} \mathrm{rifampcin/honey} \mathrm{solution} \mathrm{for} 1$ day. After feeding, females in the curing protocol were

185 given eggcards and the rifampcin/honey mixture. The emergent second and third generations

186 were likewise given rifampcin/honey solution for one day prior to eggcards and oviposition. The

187 following $\left(4^{\text {th }}\right)$ generation pupae were isolated and emerging females given E. kuehniella host

188 eggs for virgin oviposition on day 1. On day 2 the previous day's host eggs were removed and

189 placed in a $12 \times 75 \mathrm{~mm}$ glass culture tube for rearing in order to confirm the all-male offspring

190 expected following curing. A male from the same line and an eggcard were placed in the culture

191 tube containing the isolated female, and this culture was then maintained as a cured sexual line.

192 After 3 generations of mass mating, pupae from the cured population were isolated and female

193 offspring were tested again for cured status by the failure to amplify Wolbachia16S rDNA, and

194 the virgin production of only male offspring. 
196 Using the four experimental lines, novel combinations were created by horizontal transfer in the

197 laboratory by infection of each cured subline with the three alternative types of Wolbachia. A

198 superparasitization technique developed by Huigens et al. (2000) was used to horizontally

199 transfer Wolbachia from the original infected cultures into cured sublines to create novel $T$.

200 kaykai/Wolbachia combinations. All original lines were uniquely genotyped by observable

201 agarose gel band size differences using microsatellite markers specifically designed for T. kaykai

202 (Huigens 2003) to confirm successful horizontal transfer of Wolbachia. Cured and infected

203 females were allowed to oviposit on the same host (Trichoplusia ni). F1 females from

204 superparasitized hosts were genotyped and tested for Wolbachia infection status. Successful

205 horizontal transfer of Wolbachia infection was determined by production of female offspring by

206 virgin females who were identified by their nuclear genotype as having come from cured

207 sublines, thus creating new cross-infected isofemale cultures. The original lines were not re-

208 created by re-infection of cured lines. All cross-infected lines (novel infections created by

209 horizontal transfer of Wolbachia) were cultured for 20 generations prior to final fitness testing.

210 The 20 generation period was chosen to: 1) assure stable inheritance of novel Wolbachia

211 infection and 2) control for any immediate or near-immediate effects of the horizontal transfer

212 protocol on fitness.

213 Fitness test for T. kaykai-Wolbachia interaction

214 Three fitness traits, total pupae, total offspring and total female offspring, were measured for the

21516 T. kaykai-Wolbachia lines using a minimum of 35 unmated females from each line. From

216 these measurements pupal survival and offspring sex ratios were derived as proportions. To

217 control for possible Wolbachia titer differences associated with age (Jeong \& Stouthamer 2004)

218 among replicates, experimental females were derived from offspring collected on day one (the 
219 first day) of oviposition by females that were themselves sampled on day one, i.e. the first-born

220 progeny of first-born progeny. All lines in the final fitness test were assayed at the same time.

221 Pupae were isolated in $12 \times 75 \mathrm{~mm}$ glass culture tubes. Upon emergence, these isolated females

222 were given eggcards for oviposition and honey for 24 hours. Afterwards eggcards were removed

223 and replaced with a fresh eggcard and honey. Each removed eggcard was placed in a culture tube

224 and incubated for 14 days. Each female was given a total 6 eggcards, the first 5 for 24 hour

225 oviposition, while the $6^{\text {th }}$ eggcard was left with the female for 96 hours $(4 \times 24$ hours). After the

22610 day test period the experiment was stopped. All eggcards were scored for fecundity- offspring

227 reaching pupal development stage, and female offspring production, pupal survival, and

228 offspring sex ratio (female offspring/total offspring). Pupal survival was defined as the ratio

229 between the number of eclosed adult T. kaykai and total observed pupal development. Female

230 offspring production, was included as a fecundity measure to illustrate the complexity of

231 offspring production and sex ratio interactions across host and Wolbachia types.

\section{Statistical analysis.}

233 The fitness measures were used to test hypotheses related to the effect of host and Wolbachia

234 genotype; including host-Wolbachia interactions and the effect of horizontal transfer (>97

235 generations of strict co-inheritance vs. novel combinations created by horizontal transfer).

236 Fligner-Killeen tests were conducted on count data to test statistical assumptions associated with

237 homogeneity of variances for categorical variables (Conover et al. 1981).

238 To partition fitness effects between T. kaykai and Wolbachia genomes excluding any potential

239 coevolutionary effects associated with the original infection lines, generalized linear models

240 (GLMs) excluding original infections (diagonal cells, Fig. 1) were fit to each fitness measure for

241 all novel T. kaykai/Wolbachia combinations (those treatments created by horizontal transfer of 
242 Wolbachia), with Host and Wolbachia as the categorical explanatory variables. For each fitness

243 measure, two GLMs (including the effect of host type and Wolbachia type) were fitted to the

244 data, one with and one without host-Wolbachia interactions. Analysis of deviance hypothesis

245 tests were then used to determine if any significant differences existed between the two models.

246 Fecundity count data models were assumed to have a quasi-Poisson error structure (Poisson

247 distributed errors corrected for overdispersion) while offspring sex ratio (female offspring/total

248 offspring) and pupal survival (total offspring/total pupae) frequency data models were assumed

249 to have a quasi-binomial error structure (binomial distributed errors corrected for overdispersion)

250 The effects of horizontal transfer on all fitness measures were also analyzed with GLMs using

251 the categorical explanatory variables: novel infections created by horizontal transfer of

252 Wolbachia (all off-diagonal cells, Fig. 1) and original infections (all diagonal cells).

253 Nonhomogeneity of fecundity fitness variance was observed for host background, Wolbachia

254 background and horizontal transfer (comparing the novel infection lines created by horizontal

255 transfer with the original infection lines), supporting the use of generalized linear models instead

256 of classical ANOVA (aov) (Supp. Table 1).

\section{Results.}

Host Fitness Variation

259 The four host genetic backgrounds used in this experiment showed significant variation for all

260 fitness variables measured (Table 1, Figs. 2-5a). Among the novel horizontal transfer lines (all

261 off-diagonal treatments, Fig. 1) the effect of the host genetic background was significant for

262 fecundity- pupal and female production, survival, and offspring sex ratio (Table 1). The $T_{2}$ and

$263 T_{3}$ host lines produced on average about 50\% more offspring than the other two host lines (Figs.

264 2c-3c, Supp. table 2a-b for factorial results). Pupal survival for the least fit host $\left(T_{3}\right)$ was $5.2 \%$ 
265 lower than for the most fit host $\left(T_{1}\right)$, i.e. $81.7 \%$ vs. $86.9 \%$ (Fig. 4c, Supp. table 2c). The $T_{1}$ host

266 line produced fewer female offspring proportionally when compared to the other host lines.

267 Differences in proportional female offspring between host line $T_{1}$ and the other host lines

268 resulting in offspring sex ratio differences that ranged from $7 \%$ to $9 \%$ (Fig. 5c, Supp. Table $2 \mathrm{~d}$ ).

269 Taken together, relative fitness among the four host lines varied regarding the four measured

270 fitness variables, with host lines $T_{2}$ and $T_{3}$ showing overall higher fecundity, and the $T_{1}$ host line

271 with higher overall survival but lower offspring sex ratio (proportional female offspring).

\section{Wolbachia Fitness Variation.}

273 The four Wolbachia types $\left(W_{1}-W_{4}\right)$ had no significant effect on observed variation for pupal

274 fecundity and survival (Table 1, Fig. 2b, Fig. 4b). However, a significant Wolbachia effect

275 associated with female fecundity was observed $(\mathrm{p}<0.012$, Table 1$)$ with the $W_{4}$ type, which

276 produced $10.5 \%$ more female offspring than the next highest line, $W_{l}\left(W_{4}\right.$ mean $=34.2 ; W_{l}$ mean

$277=30.6$, Supp. table 2b). The influence of Wolbachia type was most clearly observed in the highly

278 significant variation among the four types for offspring sex ratio (Fig. 5b, Table 1). The higher

279 number of female offspring produced by Wolbachia type $W_{4}$ was reflected in significant

280 variation for sex ratio with type $W_{4}$ producing proportionally more female offspring than the next

281 highest line, $W_{2}\left(W_{4}\right.$ sex ratio $=0.85 ; W_{2}$ sex ratio $\left.=0.80\right)$, and the two lowest offspring sex ratio

282 Wolbachia types, $W_{1}$ and $W_{3}$, producing between $4 \%$ to $11 \%$ fewer female offspring

283 proportionally than the two highest offspring sex ratio lines $\left(W_{l}\right.$ sex ratio $=0.76 ; W_{3}$ sex ratio $=$

284 0.74) Supp. Table 2d). The fitness effects associated with variation among the Wolbachia types

285 was largely confined to significant offspring sex ratio differences, with a residual effect on the

286 overall number of female offspring.

287 Host- Wolbachia genotype fitness interactions. 
288 Fecundity fitness variation, offspring reaching pupal stage of development and female offspring

289 production, for T. kaykai was solely a function of the main host and, to a lesser extent,

290 Wolbachia type effects, with no significant host-Wolbachia interaction (Table 1, Fig. 2a, 3a,d).

291 However interaction effects were observed for pupal survival $(\mathrm{p}<0.007$, Fig. 4a,d) and strong

292 interaction effects were observed for offspring sex ratio ( $p<0.001$, Table 1, Fig. 5a,d). Unlike

293 fecundity, T. kaykai fitness regarding pupal survival and offspring sex ratio is a function of

294 strong host effects for both fitness parameters, strong Wolbachia effects for offspring sex ratio,

295 and interaction effects associated with distinct host-Wolbachia combinations.

296 Original versus Novel infection (horizontal transfer effect)

297 There were no significant effects of horizontal transfer on the number of offspring reaching the

298 pupal stage of development, female offspring production, or offspring sex ratio. Though the

299 mean fecundity values for the original infection lines (diagonal cells, Fig. 1) were higher than the

300 mean values for the horizontal transfer lines (off-diagonal cells), the differences were not

301 significant (Table 2). The only significant effect of horizontal transfer was observed for pupal

302 survival $(\mathrm{p}<0.05)$; with horizontal transfer host-Wolbachia combinations suffering a small, but

303 consistent, 1.4\% decrease in pupal survival relative to the original infection lines (Table 2; Supp.

304 Table 2c). Fitness for the novel host-Wolbachia combinations created by horizontal transfer was

305 not significantly different from the original combinations, with the exception of a slightly

306 significant negative effect on pupal survival in the horizontally transferred lines.

307 Discussion.

308 The fitness variables in which Trichogramma kaykai and Wolbachia's evolutionary interests

309 would seem to align, fecundity and survival, showed similar patterns of significant host effects, 
310 and little or no overall Wolbachia effect. There were no significant host-Wolbachia interactions

311 associated with fecundity, however a significant interaction effect for survival was observed.

312 The fitness variable that presents a potential conflict of interest for T. kaykai and Wolbachia,

313 offspring sex ratio, showed significant host, Wolbachia and interaction effects.

314 Taken together, the analysis of main effects on fitness suggests that the absence of any strong

315 variation due to the different host-associated Wolbachia strains may be a result of Wolbachia

316 genotypes having been selected to the same "good mixer" optimum, in which the influence of

317 Wolbachia on host genes optimized for reproductive fitness is attenuated. Combined with

318 significant variation across host types our observations suggest PI-Wolbachia essentially "blend

319 into" the fitness landscape of the host environment. This is consistent with expectation given that

320 with respect to reproduction and survival the interests of both the wasp and the Wolbachia are

321 aligned since higher fecundity and higher survival favors both parties. Similar results have been

322 observed for Rickettsia infections in the whitefly Bemisia tabaci, where introgression

323 experiments showed no significant variation among extranuclear genotypes (cytoplasmic

324 elements, including Rickettsia) for host fecundity and development fitness measures (Hunter et

325 al. 2016).

In contrast to the host-dependent source of fecundity and survival variation, variation in sex ratio was driven by host, Wolbachia and their interaction (Table 2). For symbioses where maternally inherited symbionts manipulate host sex ratios, the interaction between host and symbiont is characterized by conflict over control of offspring sex ratios (Werren 2011). However the extent to which control is exerted by host and symbiont is often unknown. A recent study on the $B$. tabaci-Rickettsia symbiosis, in which Rickettsia manipulate sex ratios in a female-biasing 
333 and host-symbiont interactions (Hunter et al. 2016). For infected T. kaykai females, offspring sex

334 ratios are a function of the efficiency of Wolbachia-induced gamete duplication (the means by

335 which PI Wolbachia converts male offspring to female offspring). Wolbachia-nuclear conflict

336 over offspring sex ratios predicts nuclear selection for host resistance to gamete duplication

337 efficiency would favor the production of male offspring in female-biased populations

338 (Stouthamer et al. 2001, Stouthamer et al. 2010). Host nuclear suppression of Wolbachia-

339 induced sex ratio distortion has been observed in the butterfly Hypolimnas bolina (Hornett et al.

340 2006), where sex ratio distortion takes the form of male-killing, rather than PI-Wolbachia

341 feminization and parthenogenesis-induction. Though previous analysis found no evidence of

342 suppressor alleles in the T. kaykai population (Stouthamer et al. 2001), a more detailed analysis

343 of variation for this character in T. kaykai may reveal cryptic genetic variation for suppression of

344 gamete duplication. It is possible that the titer of the bacteria may be influenced by the host

345 genome which may, in turn, influence the gamete-duplication ability of Wolbachia in a manner

346 similar to the dose-dependent effect of antibiotics on Wolbachia-infected Musicifurax uniraptor

347 sex ratios (Zchori-Fein et al. 2000). Wolbachia titer in Trichogramma pretiosum was found to

348 be positively associated with the production of infected female offspring (Lindsey \& Stouthamer

349 2017a). Wolbachia titer is associated with transmission efficiency and may be a target of

350 conflicting sex ratio selection in the T. kaykai-Wolbachia symbiosis, with T. kaykai selection for

351 less efficiency, resulting more male offspring, particularly in female-biased sex ratio distorted

352 environments.

353 Wolbachia types may vary in their gamete duplication efficiency. Schneider et al. (2013)

354 uncovered cryptic Wolbachia population heterogeneity in the European cherry fruit fly,

355 Rhagoletis cerasi, when horizontally transferred to novel hosts where subsequent fitness 
356 variation in novel host backgrounds was observed. Given the expectation of divergent

357 evolutionary optima for T. kaykai and Wolbachia, the finding of a significant interaction for

358 offspring sex ratio in our experiment was not surprising. While PI Wolbachia benefits from

359 female-biasing gamete duplication efficiency; T. kaykai nuclear genes benefit from gamete

360 duplication inefficiency and male production in female-biased sex ratio distorted populations.

361 The observed significant main and interaction effects for offspring sex ratio may be an indication

362 of cytoplasmic-nuclear genomic conflict over sex ratios in the T. kaykai-Wolbachia symbiosis.

363 One proposed resolution to this evolutionary conflict is sex ratio selection on host fertilization

364 frequencies resulting in the loss of female sexual function, the fixation of Wolbachia infection,

365 and the alignment of fitness interests favoring the transmission and gamete duplication ability of

366 Wolbachia (Stouthamer et al. 2010).

367 Although the overall effect of horizontal transfer was negative when the original and novel lines

368 were tested for fecundity and survival, with fecundity decreased by 7\% (Supp. Table 2a-b) and

369 pupal survival reduced from 0.86 to 0.84 , only survival was shown to be statistically significant

370 (Table 2). It was not possible to distinguish whether these fitness reductions were a result of

371 Wolbachia adaptation to host genomic environments subsequently disrupted by horizontal

372 transfer, or simply a result of fitness costs associated with the horizontal transfer procedure used.

373 The 20-generation incubation period for horizontally transferred Wolbachia lines in this

374 experiment was intended to control for any immediate fitness effects associated with the

375 horizontal transfer. But it should be noted that negative fitness effects associated with horizontal

376 transfer of Arsenophonus inherited symbionts have been observed in aphids (Russell \& Moran

377 2005), where a four generation horizontal transfer incubation period was used. Horizontal

378 transfer to novel host backgrounds and introgression experiments have shown Wolbachia is 
379 capable of rapid adaptation to novel host environments (Newton and Sheehan 2015) and

380 significant phenotypic shifts (Fujii et al. 2001, McGraw et al. 2002, Sasaki et al. 2002, Jaenike

381 2007). The dynamism of host-Wolbachia interactions has been exploited in transfection

382 experiments with Wolbachia strain wMelPop in which transfer to novel hosts has resulting in

383 immune activation that limits competency of cohabiting symbionts like viruses and filarial

384 nematodes (Kambris et al. 2009, Moreira et al. 2009).

385 The observed pupal survival costs associated with horizontal transfer may be the result of

386 dynamics associated with T. kaykai-Wolbachia genomic cooperation (Herre et al. 1999; Rand et

387 al. 2004; Vautrin \& Vavre 2009). In other words, coadaptation between host and Wolbachia in

388 the original infected lines may have resulted in reduced survival costs for T. kaykai, and such

389 coadaptation may have not had time to occur in the novel host-Wolbachia combinations created

390 by horizontal transfer. How T. kaykai-Wolbachia coadaptation for pupal survival (or fecundity)

391 would take place is unknown, but it is unlikely in our experiment that changes would take place

392 in the wasp genome since the lines used were $100 \%$ homozygous and 100 generations would be

393 too short for an appreciable number of mutations to accumulate. Consequently, changes would

394 have to take place in the Wolbachia genome or population. Per wasp egg per generation around

395 400-1000 Wolbachia cells are passed on from mother to her offspring (Stouthamer \& Werren

396 1993). Wolbachia adaptation to a host environment has been observed in the Wolbachia strain

397 known as wMelPop when serial passaged in novel host cell culture (McMeniman et al. 2008); an

398 environment not unlike the strict cyto-nuclear inheritance of unmated PI Wolbachia-infected

399 species. Newton and Sheehan (2015) found that Wolbachia titer adapted to novel Drosophila

400 melanogaster nuclear backgrounds in as little as three generations. 
401 Alternatively, Wolbachia-mitochondria interactions may play a role in the observed fitness

402 deductions observed for the horizontally transferred lines. Recent results with Trichogramma

403 pretiosum showed that nuclear introgression of novel genetic background appeared to have no

404 negative fecundity fitness effects (Lindsey \& Stouthamer 2017b). However, horizontal transfer

405 of Wolbachia was not possible with T. pretiosum, unlike here where Wolbachia were

406 experimentally transferred to novel host nuclear and mitochondrial backgrounds. Future

407 experiments in which horizontal transfer and nuclear introgression are applied to the same

408 system would be useful in determining potential T.kaykai-Wolbachia coadaptation.

\section{Conclusion}

Wolbachia in the T. kaykai population would appear to be good "mixers" in regard to shared

fitness interests, exhibiting similar mean fitness values across T. kaykai backgrounds for

fecundity and survival. Though Wolbachia may not increase fitness for T. kaykai (Russell et al.

2016, Tagami et al. 2001), variation in survival and reproduction among the infected population

host backgrounds. The strong main and interaction effects observed for offspring sex ratios

417 for a cytoplasmic sex ratio distorter like PI-Wolbachia, and may be a signature of Wolbachianuclear conflict. Support for the good mixer-evolutionary conflict hypothesis has been demonstrated in introgression experiments with sex ratio-distorting Rickettsia infected population of the whitefly, B. tabaci, where shared interest traits, such as fecundity, are

422 sex ratio, are influenced by host-symbiont interactions (Hunter et al. 2016); like the results found 
424 be useful in determining the general applicability of a conflict hypothesis in the T.kaykai-

425 Wolbachia symbiosis.

426 Acknowledgments: Thanks to Phailak Rangsingharn for help with counting and maintaining

427 wasps. Thanks to Debra Hughson with the United States National Park Service, Mojave National

428 Preserve.

429

430

431

432

433

434

435

436

437

438

439

440

441 
443

444

445

446

447

448

449

450

451

452

453

454

455

456

457

458

459

460

461

462

463

464

465

466

467

468

469

470

471

472

473

474

475

476

Arakaki N, Miyoshi T, Noda H (2001) Wolbachia-mediated parthenogenesis in the predatory thrips Fanklintothrips vespiformis (Thysanoptera : Insecta). Proc. R. Soc. B Biol. Sci. 268:10111016.

Baldo L, Dunning Hotopp JC, Jolley KA, Bordenstein SR, Biber SA, Choudhury RR, Hayashi C, Maiden MCJ, Tettelin H, Werren JH (2006) Multlocus sequence typing system for the endosymbiont Wolbachia pipientis. Appl. Environ. Microb. 72(11): 7098-7110.

Conover WJ, Johnson ME, Johnson MM (1981). A comparative study of tests for homogeneity of variances, with applications to the outer continental shelf bidding data. Technometrics 23: 351-361. doi: 10.2307/1268225. Cosmides LM, Tooby J (1981) Cytoplasmic inheritance and intragenomic conflict. J. Theor. Biol. 89:83-129.

Dowling DK, Friberg U, Hailer F, Arnqvist G (2007) Intergenomic epistasis for fitness: Withinpopulation interactions between cytoplasmic and nuclear genes in Drosophila melanogaster. Genetics 175:235-244.

Fujii Y, Kageyama D, Hoshizaki S, Ishikawa H, Sasaki T (2001) Transfection of Wolbachia in Lepidoptera: the feminizer of the adzuki bean borer Ostrinia scapulalis causes male killing in the Mediterranean flour moth Ephestia kuehniella. Proc. Biol. Sci. 268: 855-859.

Herre EA, Knowlton N, Mueller UG, Rehner SA (1999) The evolution of mutualisms: exploring the paths between conflict and cooperation. Trends Ecol. Evol. 14: 49-53.

Hohmann CL, Luck RF, Stouthamer R (2001) Host deprivation effect on reproduction and survival of Wolbachia-infected and uninfected Trichogramma kaykai (Hymenoptera:

Trichogrammatidae). Neotrop. Entomol. 30: 601-605.

Hornett EA, Charlat S, Duplouy AMR, Davies N, Roderick GK, Wedell N, Hurst GDD (2006)

Evolution of male-killer suppression in a natural population. PLoS Biol 4(9): e283.

Huigens ME, Luck RF, Klaassen RHG, Maas MFPM, Timmermans MJTN, Stouthamer R (2000) Infectious parthenogenesis. Nature 405:178-179.

Huigens ME (2003) On the evolution of Wolbachia-induced parthenogenesis in Trichogramma wasps. Wageningen: Entomology.Wageningen University. 183p.

Huigens ME, Hohmann CL, Luck RF, Gort G, Stouthamer R (2004) Reduced competitive ability due to Wolbachia infection in the parasitoid wasp Trichogramma kaykai. Entomol. Exp. Appl. 110: 115-123.

Huigens ME, Stouthamer R (2003) Parthenogenesis associated with Wolbachia. In Bourtzis K, Miller TA, editors. Insect Symbiosis. Boca Raton: CRC Press. pp. 247-266.

Hunter MS, Asiimwe P, Himler AG, Kelly SE (2016) Host nuclear genotype influences phenotype of a conditional mutualist symbiont. J. Evol. Biol. 30: 141-149. 
477 Jaenike J (2007) Spontaneous emergence of a new Wolbachia phenotype. Evolution 61: 22444782252.

479 Jeong G, Stouthamer R (2004) Genetics of female functional virginity in the parthenogenesis 480 Wolbachia infected parasitoid wasp Telenomus nawai (Hymenoptera:Scelionidae). Heredity 481 94:402-407.

482 Kambris Z, Cook PE, Phuc HK, Sinkins SP (2009) Immune activation by life-shortening 483 Wolbachia and reduced filarial competence in mosquitoes. Science 326(5949): 134-136

484

485

486

487

488

489

490

491

492

493

494

495

496

497

498

499

500

501

502

503

504

505

506

507

508

509

510

511

512

513

Kremer N, Charif D, Henri H, Bataille M, Prevost G, Kraaijeveld K, Vavre F (2009) A new case of Wolbachia dependence in the genus Asobara: evidence for parthenogenesis-induction in Asobara japonica. Heredity 103:248-256.

Lindsey ARI, Stouthamer R (2017a) Penetrance of symbiont-mediated parthenogenesis is driven by reproductive rate in a parasitoid wasp. PeerJ 5:e3505; DOI 10.77171/peerj.3505.

Lindsey ARI, Stouthamer R (2017b) The effects of outbreeding on a parasitoid wasp fixed for infection with a parthenogenetic-inducing Wolbachia symbiont. Heredity 119:411-417.

McGraw EA, Merrit DJ, Droller JN, O’Neill SL (2002) Wolbachia density and virulence attenuation after transfection into a novel host. Proc. Natl. Acad. Sci. USA 99: 2918-2923.

McMeniman CJ, Lane AM, Fong AWC, Voronin DA, Iturbe-Ormaetxe I, Yamada R, McGraw EA, O'Neill SL (2008) Host adaptation of a Wolbachia strain after long-term serial passage in mosquito cell lines. Appl. Environ. Microbiol. 74(22): 6963-6969.

Moreira LA, Iturbe-Ormaetxe I, Jeffery JA, Lu G, Pyke AT, Hedges LM, Rocha BC, HallMendelin S, Day A, Riegler M, Hugo LE, Johnson KN, Kay BH, McGraw EA, van den Hurk AF, Ryan PA, O'Neill SL (2009) A Wolbachia symbiont in Aedes aegypti limits infection with Dengue, Chikungunya, and Plasmodium. Cell 139: 1268-1278.

Miura K, Tagami Y (2004) Comparison of life history characters of arrhenotokous and Wolbachia-associated thelytokous Trichogramma kaykai Pinto and Stouthamer (Hymenoptera: Trichogrammatidae). Ann. Entomol. Soc. Am. 97:765-769.

Newton ILG, Sheehan KB (2015) Passage of Wolbachia pipientis through mutant Drosophila melanogaster induces phenotypic and genomic changes. Appl. Environ. Microb. 81(3): 10321037.

Pannebakker BA, Schidlo NS, Boskamp GJF, Dekker L, Van Dooren TJM, Beukeboom LW, Zwaan BJ, Brakefield PM, Van Alphen JJM (2005) Sexual functionality of Leptipolina clavipes (Hymenoptera: Figitidae) after reversing Wolbachia-induced parthenogenesis. J. Evol. Biol. 18: 1019-1028.

Pfarr KM, Hoerauf A (2007) A niche for Wolbachia. Trends Parasitol. 23:5-7.

Pinto JD, Stouthamer R, Platner GR (1997) A new species of Trichogramma (Hymenoptera, Trichogrammaditae) from the Mojave Desert of California as determined by morphological, reproductive, and molecular data. P. Entomol. Soc. Wash. 99:238-247. 
514 Rand DM, Haney RA, Fry AJ (2004) Cytonuclear coevolution: the genomics of cooperation.

515 Trends Ecol. Evol. 19:645-653.

516 Russell JA, Moran NA, (2005) Horizontal transfer of bacterial symbionts: heritability and fitness 517 effects in a novel aphid host. Appl. Environ. Microbiol. 71(12): 7987-7984.

518 Russell JE (2008) The ecological and evolutionary consequences of Wolbachia infection in 519 Trichogramma species. Riverside: Entomology.University of California. 147p.

520 Russell JE, Stouthamer R (2011) The genetics and evolution of obligate reproductive parasitism

521 in Trichogramma pretiosum infected with parthenogensis-inducing Wolbachia. Heredity 106:58-

52267.

523 Russell JE, Saum M, Burgess V, Bollavaram K, Donnell T (2016) Influence of parthenogenesis-

524 inducing Wolbachia infection and sexual mode on Trichogramma kaykai

525 (Hymenoptera:Trichogrammatidae) fitness. Ann. Entomol. Soc. Am. 110:263-268.

526 Sasaki T, Kubo T, Ishikawa H (2002) Interspecific transfer of Wolbachia between two

527 lepidopteran insects expressing cytoplasmic incompatibility: a Wolbachia variant naturally

528 infecting Cadra cautella causes male killing in Ephestia keuhniella. Genetics 162(3): 1313-1319.

529 Schneider DI, Reigler M, Arthofer W, Mercot H, Stauffer C, Miller WJ (2013) Uncovering

530 Wolbachia Diversity upon Artificial Host Transfer. PLoS ONE 8(12): e82402.

531

532

533

Stouthamer R, Luck RF, Hamilton WD (1990) Antibiotics cause parthenogenetic Trichogramma (Hymenoptera, Trichogrammatidae) to revert to sex. Proc. Natl. Acad. Sci. USA. 87:2424-2427.

534 Stouthamer R, Werren JH (1993) Microbes associated with parthenogenesis in wasps of the 535 genus Trichogramma. J. Invertebr. Pathol. 61:6-9.

536 Stouthamer R, Kazmer DJ (1994) Cytogenetics of microbe-associated parthenogenesis and its 537 consequences for gene flow in Trichogramma wasps. Heredity 73:317-327.

538 Stouthamer R, van Tilborg M, de Jong JH, Nunney L, Luck RF (2001) Selfish element maintains 539 sex in natural populations of a parasitoid wasp. Proc. R. Soc. B Biol. Sci. 268:617-622.

540 Stouthamer R, Russell JE, Vavre F, Nunney L (2010) Intragenomic conflict in populations

541 infected by parthenogenesis inducing Wolbachia ends with irreversible loss of sexual

542 reproduction. BMC Evol. Biol. 10:229.

543 Tagami Y, Miura K, Stouthamer R (2001) How does infection with parthenogenesis-inducing 544 Wolbachia reduce the fitness of Trichogramma? J. Invertebr. Pathol. 78:267-271.

545 Tagami Y, Miura K, Stouthamer R (2002) Positive effect of fertilization on the survival rate of 546 immature stages in a Wolbachia-associated thelytokous line of Trichogramma deion and $T$.

547 kaykai. Entomol. Exp. Appl. 105:165-167.

548 Turelli M (1994) Evolution of incompatibility-inducing microbes and their hosts. Evolution 48:

$549 \quad 1500-1513$. 
550 Vautrin E, Vavre F (2009) Interactions between vertically transmitted symbionts: cooperation or 551 conflict? Trends Microbiol. 17(3): 95-99.

552 Wade MJ, Goodnight CJ (2006) Cyto-nuclear epistasis: Two-locus random genetic drift in

553 hermaphroditic and dioecious species. Evolution 60:643-659.

554 Weeks AR, Breeuwer JAJ (2001) Wolbachia-induced parthenogenesis in a genus of

555 phytophagous mites. Proc. R. Soc. B Biol. Sci. 268:2245-2251.

556 Weeks AR, Turelli M, Harcombe WR, Reynolds KT, Hoffmann AA (2007) From parasite to

557 mutualist: Rapid evolution of Wolbachia in natural populations of Drosophila. PLoS Biol 5(5):

558 e114.

559 Werren JH, Windsor D, Guo LR (1995) Distribution of Wolbachia among neotropical

560 arthropods. Proc. R. Soc. B Biol. Sci. 262:197-204.

561 Werren JH (1997) Biology of Wolbachia. Annu. Rev. Entomol. 42:587-609.

562 Werren JH (2011) Selfish genetic elements, genetic conflict, and evolutionary innovation. Proc.

563 Natl. Acad. Sci. USA 108:10863-10870.

564 Zchori-Fein E, Gottlieb Y, Coll M (2000) Wolbachia Density and Host Fitness Components in

565 Muscidifurax uniraptor (Hymenoptera: Pteromalidae) J. Invertebr. Pathol. 75:267-272

566

567

568

569

570

571

572

573

574

575

576 
Figure 1

4x4 Factorial design for horizontal transfer of Wolbachia in Host Trichogramma kaykai.

The shaded diagonal represents the original infections from field-caught $T$. kaykai females; all off-diagonals represent novel infections created by horizontal transfer of Wolbachia.

\section{Host type}

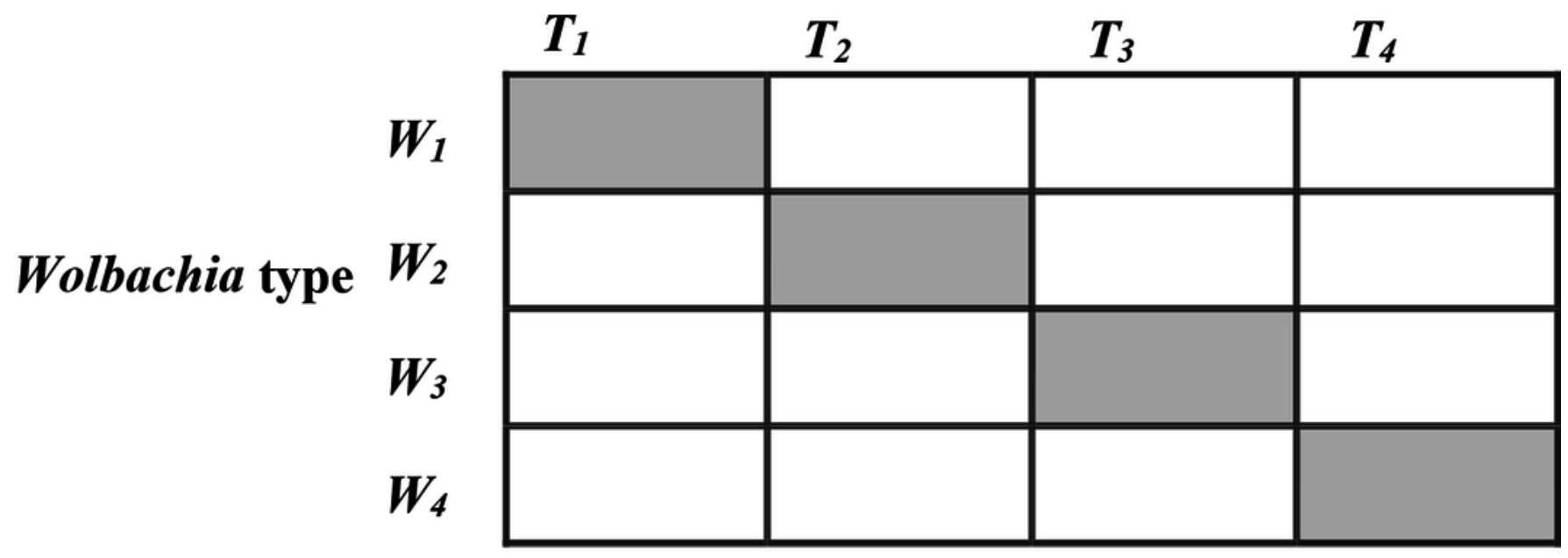




\section{Figure 2 (on next page)}

Total Pupae: main effects and 2-way interactions.

a) Host Interaction for Total Pupae: variation in total offspring reaching pupal developmental stage across the four T. kaykai host strains (T1-T4) conditioned on the infected Wolbachia types (W1-W4) is shown; b) Wolbachia Variation for Total Pupae: box plots show median values and variation across Wolbachia types for total pupae. Bold central lines represent median values, box limits represent the interquartile range (Q3-Q1), with whisker extensions to data points not more than $1.5 \mathrm{x}$ interquartile range. Points outside whiskers are deemed outliers; c) Host Variation for Total Pupae: box plots show median values and variation across T. kaykai host strains for total pupae; d) Wolbachia Interaction for Total Pupae: variation in total offspring reaching pupal developmental stage across the four Wolbachia types (W1-W4) conditioned on the infected T. kaykai host strains (T1-T4) is shown. 
(a) Host Interaction for Tota! Pupae

75

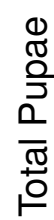

25

(c) Host Variation for Total Pupae

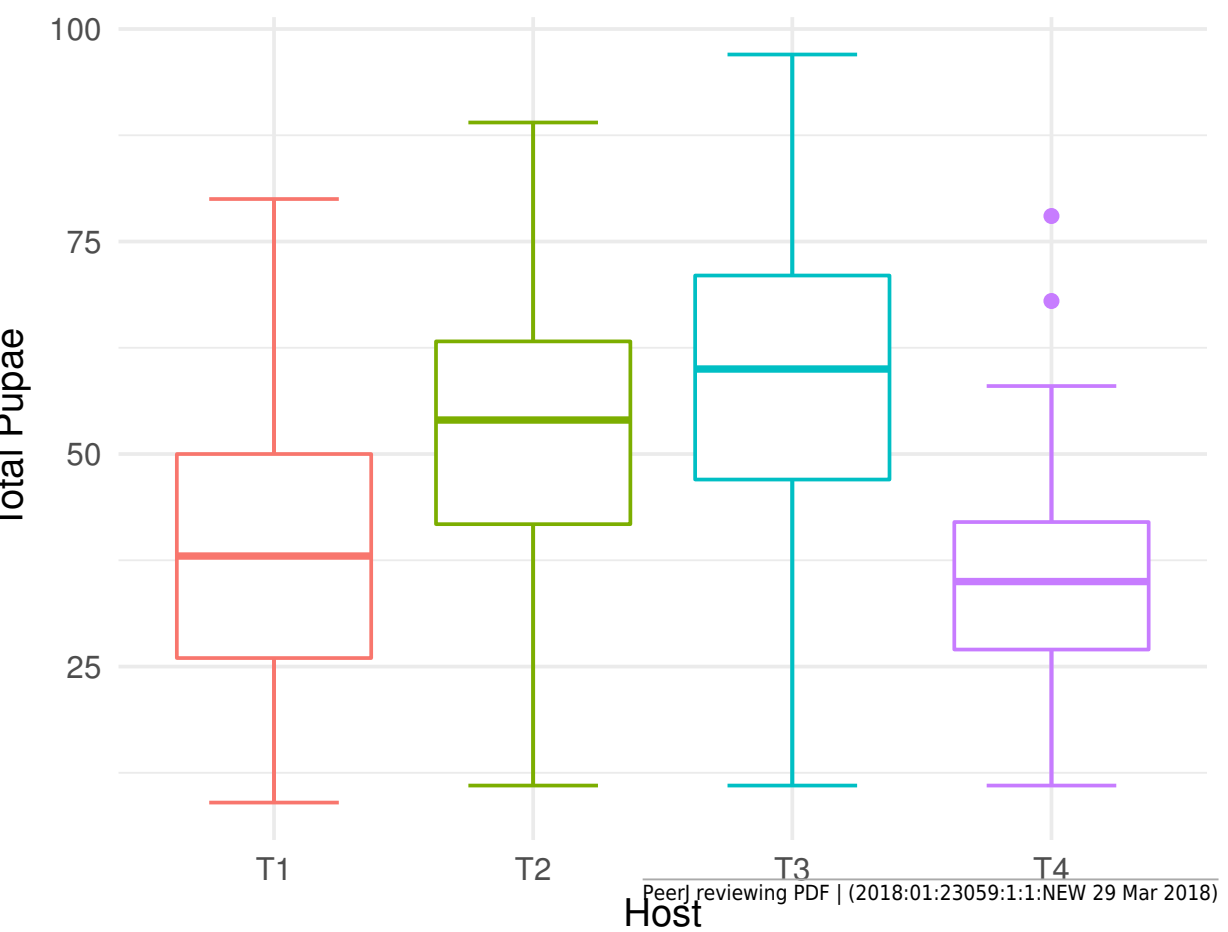

(b) Wolbachia Variation for Total Pupae

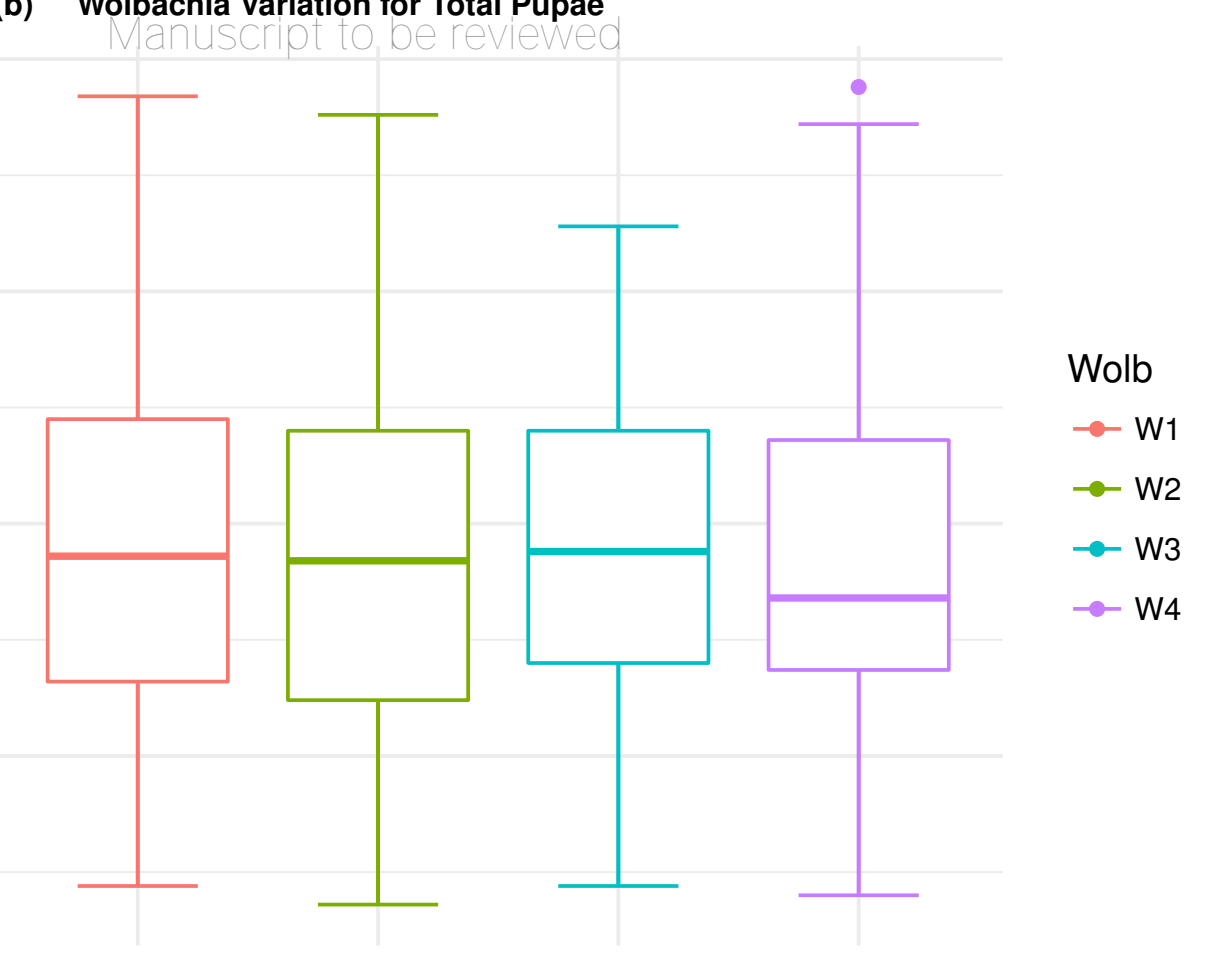

(d) Wolbachia Interaction for Total Pupae
Host

$\rightarrow \mathrm{T} 1$

$\rightarrow \mathrm{T} 2$

$\rightarrow \mathrm{T} 3$

$\multimap$ T4 


\section{Figure 3 (on next page)}

Total Female: main effects and 2-way interactions.

a) Host Interaction for Total Female: variation in total female offspring across the four T. kaykai host strains (T1-T4) conditioned on the infected Wolbachia types (W1-W4) is shown; b) Wolbachia Variation for Total Female: box plots show median values and variation across Wolbachia types for total female offspring. Bold central lines represent median values, box limits represent the interquartile range (Q3-Q1), with whisker extensions to data points not more than $1.5 \mathrm{x}$ interquartile range. Points outside whiskers are deemed outliers; c) Host Variation for Total Female: box plots show median values and variation across T. kaykai host strains for total female offspring; d) Wolbachia Interaction for Total Female: variation in total female offspring across the four Wolbachia types (W1-W4) conditioned on the infected T. kaykai host strains (T1-T4) is shown. 
(a) Host Interaction for Total Fomale

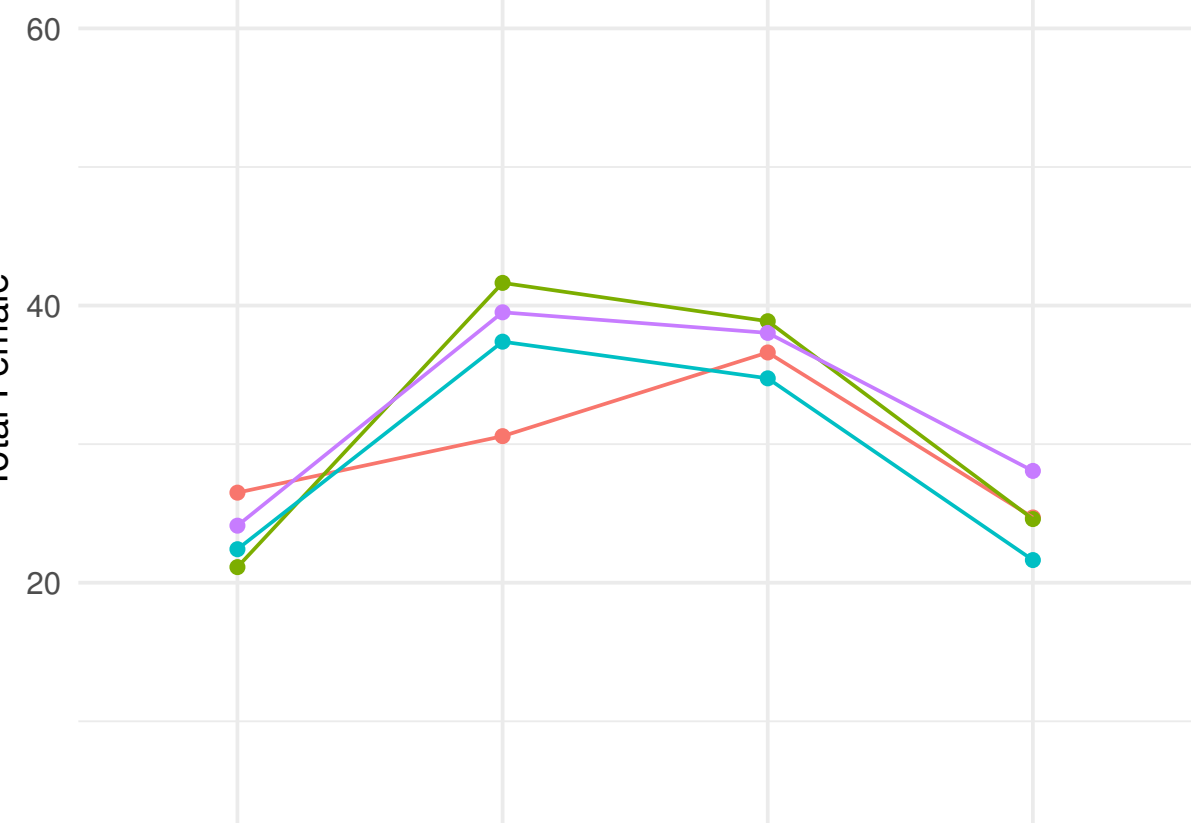

(c) Host Variation for Total Female

60

20

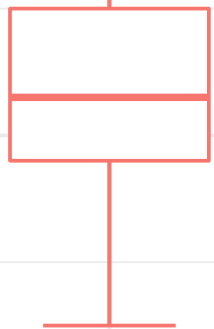

$\mathrm{T} 1$

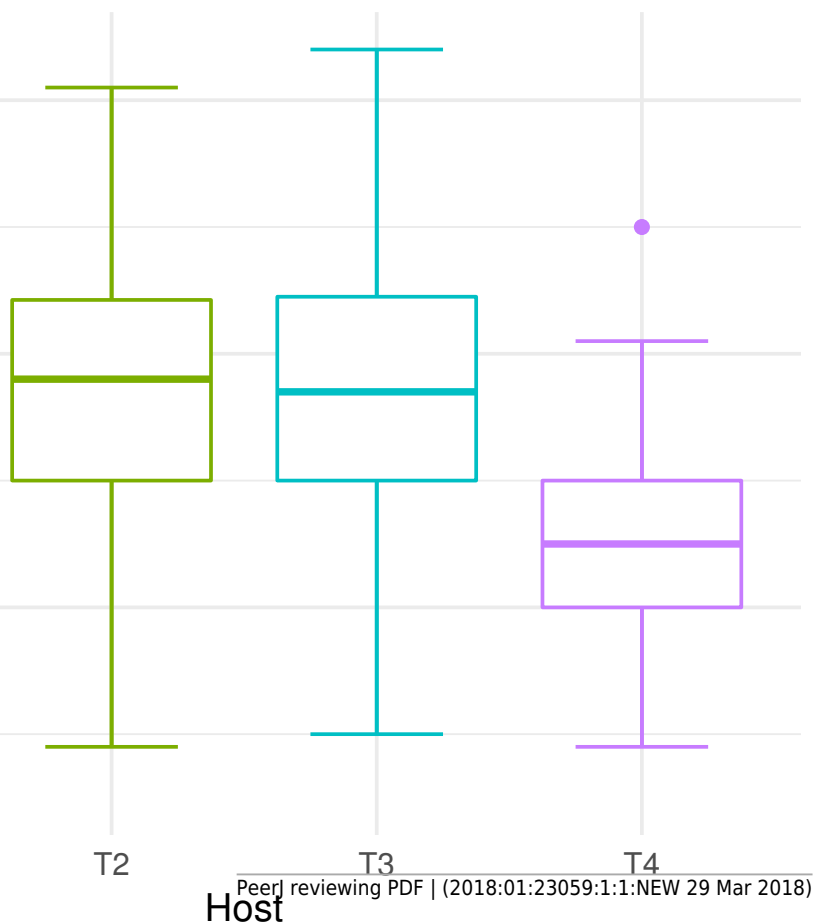

(b) Wolbachia Variation for Total Female

Manuscript to be reviewed

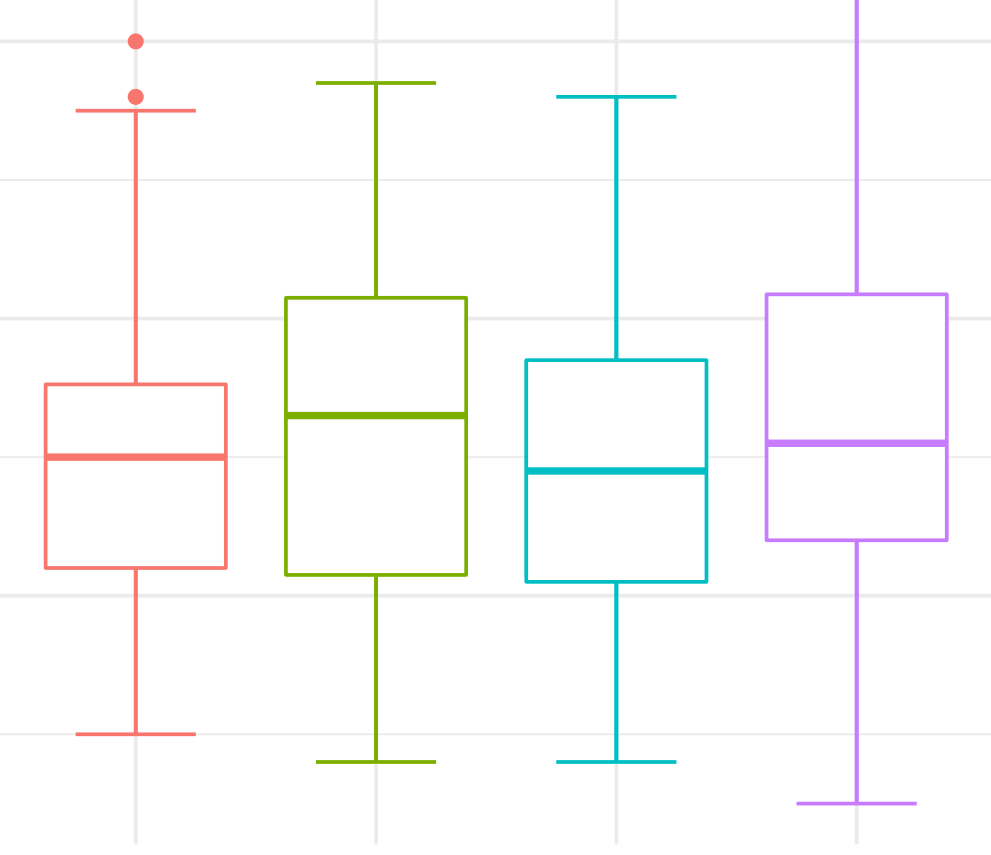

(d) Wolbachia Interaction for Total Female
Wolb

$\rightarrow-W 1$

$\rightarrow$ W2

$\rightarrow-W 3$

$\rightarrow$ W4

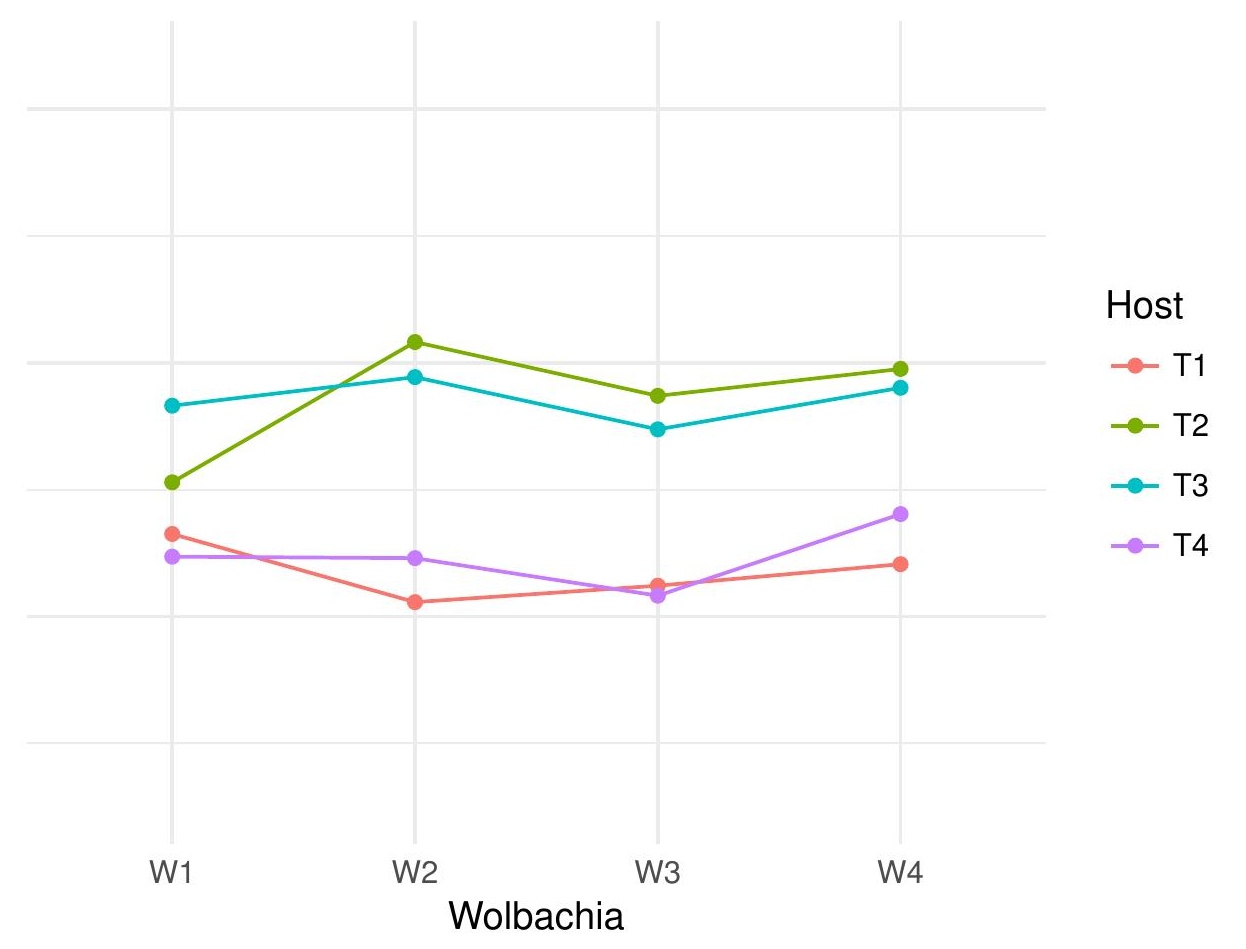




\section{Figure 4 (on next page)}

Pupal survival: main effects and 2-way interactions.

a) Host Interaction for Pupal Survival: variation in survival for offspring reaching pupal developmental stage across the four T. kaykai host strains (T1-T4) conditioned on the infected Wolbachia types (W1-W4) is shown; b) Wolbachia Variation for Pupal Survival: box plots show median values and variation across Wolbachia types for pupal survival. Bold central lines represent median values, box limits represent the interquartile range (Q3-Q1), with whisker extensions to data points not more than $1.5 \mathrm{x}$ interquartile range. Points outside whiskers are deemed outliers; c) Host Variation for Pupal Survival: box plots show median values and variation across T. kaykai host strains for pupal survival; d) Wolbachia Interaction for Pupal Survival: variation in survival for offspring reaching pupal developmental stage across the four Wolbachia types (W1-W4) conditioned on the infected T. kaykai host strains (T1-T4) is shown. 


\section{Figure 5 (on next page)}

Sex ratio: main effects and 2-way interactions.

a) Host Interaction for Sex Ratio: variation in offspring sex ratio (female/total) across the four T. kaykai host strains (T1-T4) conditioned on the infected Wolbachia types (W1-W4) is shown; b) Wolbachia

Variation for Sex Ratio: box plots show median values and variation across Wolbachia types for offspring sex ratio. Bold central lines represent median values, box limits represent the interquartile range (Q3-Q1), with whisker extensions to data points not more than $1.5 \times$ interquartile range. Points outside whiskers are deemed outliers; c) Host Variation for Sex Ratio: box plots show median values and variation across $T$. kaykai host strains for offspring sex ratio; d) Wolbachia Interaction for Sex ratio: variation in offspring sex ratio across the four Wolbachia types (W1-W4) conditioned on the infected T. kaykai host strains (T1-T4) is shown. 


\section{Table $\mathbf{1}$ (on next page)}

Results of Generalized Linear Models (GLMs)

Generalized linear model results for the four fitness traits evaluated are shown below with significant $p$ values for host, Wolbachia, and host-Wolbachia interaction effects. The significance values in the interactions row refers to the analysis of deviance results comparing GLM models with and without interactions. 
Table 1. Results of Generalized Linear Models (GLMs) for the four fitness traits evaluated are shown below with significant $\mathrm{p}$ values for host, Wolbachia, and host-Wolbachia interaction effects. The significance values in the interactions row refers to the Analysis of Deviance results comparing GLM models with and without interactions.

\begin{tabular}{|l|l|l|l|l|}
\hline & $\begin{array}{l}\text { Fecundity- } \\
\text { pupae }\end{array}$ & $\begin{array}{l}\text { Fecundity- } \\
\text { female }\end{array}$ & Pupal survival & Sex ratio \\
\hline Intercept & $<0.001^{* * *}$ & $<0.001^{* * *}$ & $<0.001^{* * *}$ & 0.288 \\
\hline Host (T2) & $<0.001^{* * *}$ & $<0.001 * * *$ & $0.021^{*}$ & $0.009^{* *}$ \\
Host (T3) & $<0.001^{* * *}$ & $<0.001^{* * *}$ & $<0.001 * * *$ & $<0.001^{* * *}$ \\
Host (T4) & 0.354 & 0.157 & 0.208 & $<0.001^{* * *}$ \\
\hline Wolbachia (W2) & 0.603 & 0.159 & 0.330 & $0.001^{* *}$ \\
Wolbachia (W3) & 0.243 & 0.276 & 0.160 & 0.150 \\
Wolbachia (W4) & 0.605 & $0.011^{*}$ & 0.084 & $<0.001^{* * *}$ \\
\hline Host*Wolbachi & 0.481 & 0.061 & $0.007^{* *}$ & $<0.001^{* * *}$ \\
$\boldsymbol{a}$ & & & & \\
\hline
\end{tabular}




\section{Table 2 (on next page)}

Mean fitness values and results of GLMs testing the effect of experimental horizontal transfer.

The mean values and results of GLMs testing the effect of experimental horizontal transfer of Wolbachia in T. kaykai on fitness traits are shown below. Horz. transfer refers to mean values for experimental treatments created by horizontal transfer of Wolbachia. Original refers to mean values for cultures from which horizontally transferred Wolbachia were derived. 
Table 2. The mean values and results of GLMs testing the effect of experimental horizontal transfer of Wolbachia in T. kaykai on fitness traits are shown below. Horz. transfer refers to mean values for experimental treatments created by horizontal transfer of Wolbachia. Original refers to mean values for cultures from which horizontally transferred Wolbachia were derived.

\begin{tabular}{|l|l|l|l|}
\hline & Horz. transfer & Original & p value \\
\hline Fecundity-pupae & 45.8 & 49.0 & 0.098 \\
\hline Fecundity-female & 30.4 & 32.7 & 0.061 \\
\hline Survival & 0.84 & 0.86 & $0.041^{*}$ \\
\hline Sex ratio & 0.79 & 0.78 & 0.553 \\
\hline
\end{tabular}

